\title{
Intestinal Microbial Dysbiosis in Beagles Naturally Infected with Canine Parvovirus ${ }^{\text {[S }}$
}

\author{
Jun Seok Park ${ }^{1 \dagger}$, Robin B. Guevarra ${ }^{2 \dagger}$, Bo-Ra Kim², Jun Hyung Lee ${ }^{2}$, Sun Hee Lee ${ }^{2}$, Jae Hyoung Cho ${ }^{2}$, Hyeri Kim ${ }^{2}$, \\ Jin Ho Cho ${ }^{3}$, Minho Song ${ }^{4}$, Ju-Hoon Lee ${ }^{5}$, Richard E. Isaacson ${ }^{6}$, Kun Ho Song ${ }^{1 *}$, and Hyeun Bum Kim ${ }^{2 *}$ \\ ${ }^{1}$ College of Veterinary Medicine, Chungnam National University, Daejeon 34134, Republic of Korea \\ ${ }^{2}$ Department of Animal Resources Science, Dankook University, Cheonan 31116, Republic of Korea \\ ${ }^{3}$ Division of Food and Animal Sciences, Chungbuk National University, Cheongju 28644, Republic of Korea \\ ${ }^{4}$ Division of Animal and Dairy Science, Chungnam National University, Daejeon 34134, Republic of Korea \\ ${ }^{5}$ Department of Food Science and Biotechnology, Institute of Life Science and Resources, Kyung Hee University, Yongin 17104, Republic of Korea \\ ${ }^{6}$ Department of Veterinary and Biomedical Sciences, University of Minnesota, St. Paul, MN 551058, USA
}

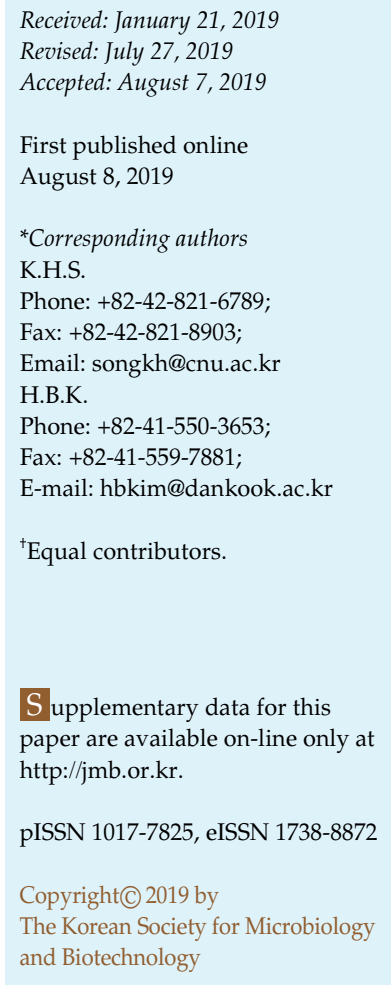

Canine parvoviral enteritis (PVE) is an important intestinal disease of the puppies; however, the potential impact of the canine parvovirus (CPV) on the gut microbiota has not been investigated. Therefore, the aim of this study was to evaluate the gut microbial shifts in puppies naturally infected with CPV. Fecal samples were collected from healthy dogs and those diagnosed with PVE at 4, 6, 8, and 12 weeks of age. The distal gut microbiota of dogs was characterized using Illumina MiSeq sequencing of the bacterial 16S rRNA genes. The sequence data were analyzed using QIIME with an Operational Taxonomic Unit definition at a similarity cutoff of $97 \%$. Our results showed that the CPV was associated with significant microbial dysbiosis of the intestinal microbiota. Alpha diversity and species richness and evenness in dogs with PVE decreased compared to those of healthy dogs. At the phylum level, the proportion of Proteobacteria was significantly enriched in dogs with PVE while Bacteroidetes was significantly more abundant in healthy dogs $(p<0.05)$. In dogs with PVE, Enterobacteriaceae was the most abundant bacterial family accounting for $36.44 \%$ of the total bacterial population compared to only $0.21 \%$ in healthy puppies. The two most abundant genera in healthy dogs were Prevotella and Lactobacillus and their abundance was significantly higher compared to that of dogs with PVE $(p<0.05)$. These observations suggest that disturbances of gut microbial communities were associated with PVE in young dogs. Evaluation of the roles of these bacterial groups in the pathophysiology of PVE warrants further studies.

Keywords: Gut microbiota, canine parvovirus, dogs, 16S rRNA

\section{Introduction}

Canine parvovirus (CPV) has been considered one of the most important pathogenic viruses in dogs since it is the causative agent of acute hemorrhagic enteritis leading to severe damage of the intestinal barrier [1, 2]. In young dogs, CPV may manifest as myocarditis resulting in the rapid development of congestive heart failure, heart arrhythmias, and sudden death [3]. CPV is an important cause of high morbidity (100\%). In addition, CPV is known to cause up to $10 \%$ mortality in adult dogs and $91 \%$ mortality in young dogs [1]. A recent study showed that dogs who survive CPV infection have a higher risk of developing chronic gastrointestinal disease [2].

The advent of next-generation sequencing of 16S rRNA genes to characterize bacterial communities has considerably 
improved our understanding of host-microbe interactions [4-7]. This technique has been applied to describe the microbial community composition and functional capacity of the gastrointestinal microbiome in dogs [8,9]. As such, recent studies have utilized sequencing of the 16S rRNA gene to determine the composition of bacterial communities in dogs with diseases including nasal disease [4], inflammatory bowel disease and intestinal lymphoma [10], food-responsive diarrhea [11], and canine pyometra [12]. However, the potential impact of the canine parvovirus $(\mathrm{CPV})$ on the gut microbiota has not been investigated. In addition, the role of bacterial communities in the pathophysiology of canine parvovirus enteritis (PVE) is not known. To date, there have been no studies evaluating shifts in the composition of the gut microflora of dogs with PVE using next-generation sequencing. Therefore, the goal of this study is to compare the intestinal microbiota of healthy dogs with that of dogs naturally infected with CPV.

\section{Materials and Methods}

\section{Animals and Diagnosis of Canine Parvoviral Enteritis}

All animal protocols were performed according to the Guidelines for the Care and Use of Laboratory Animals of Chungnam National University (No. CNU00922). Two litters of beagles were enrolled in this study including four healthy dogs and four dogs naturally infected with CPV. Each litter of beagles was separately housed in different experimental facilities at the College of Veterinary Medicine, Chungnam National University, Korea. All dogs were fed breast milk until three weeks of age and a commercial diet (Royal Canin MINI Starter Mother \& Babydog, Royal Canin, France) after weaning at 4 weeks of age. One litter of beagles was naturally infected with CPV and diagnosed as PVE at 6 weeks of age. We speculated that the CPV disease outbreak in one litter of beagles was transmitted via indirect contact with animal care personnel contaminated with canine parvovirus. Upon exposure to the CPV, the puppies normally show the clinical signs after a 3-7 day incubation period [1]. Diagnostic criteria for PVE includes complete blood count (CBC) (ADVIA 2120i, Siemens, Germany), serum chemistry profiles (BS-330, Mindray, China) and electrolytes (Easylyte Plus, Medica, USA). The PVE was diagnosed by observing the clinical signs and detection of parvovirus antigen in feces using a commercially available ELISA kit, the SNAP Parvo Test (IDEXX Laboratories, USA). Dogs naturally infected with CPV were given an intravenous infusion of Hartmann's solution and 5\% glucose (Daihan, Korea), tramadol (3 5 mg/kg, Shinpoong, Korea) as an analgesic, and ondansetrone (0.1 0.5 mg/kg, Daewoong, Korea) as an antiemetic. Granulocyte colony stimulating factor (G-CSF) $(5 \mathrm{mg} / \mathrm{kg}$, CJ Healthcare, Korea) was injected subcutaneously in CPV-infected puppies for the reproduction of damaged intestinal mucosal barrier. None of the dogs received antibiotics throughout the experiment. At week 6, ultrasonographic examination was performed on dogs infected with CPV. For imaging, the puppies were positioned in dorsal recumbency and a water-soluble coupling gel was applied liberally to the ventral abdomen to permit sound conduction.

\section{Fecal Sample Collection and DNA Extraction}

Prior to CPV exposure, fecal samples from both litters of beagles were collected from 4 weeks of age using sterile fecal swabs. At six weeks of age, after natural CPV infection was confirmed for one litter of beagles, we collected fecal samples from both healthy and sick puppies at 2-week intervals. Fecal samples were stored at $-80^{\circ} \mathrm{C}$ until DNA extraction. Total DNA representing the fecal microbial communities was extracted from $200 \mathrm{mg}$ of feces per sample using the QIAamp Fast DNA Stool Mini Kit (QIAGEN, Germany) according to the manufacturer's instructions. Cell lysis was performed by bead-beating the samples twice for $2 \mathrm{~min}$ at $300 \mathrm{rpm}$, with an incubation period of $5 \mathrm{~min}$ in a water bath at $70^{\circ} \mathrm{C}$ between beatings [13]. The concentrations of DNA were measured using a Colibri Microvolume Spectrometer (Titertek, Germany) and samples with the ratio of the absorbance at 260 and $280 \mathrm{~nm}$ of $1.80-2.15$ were processed further.

\section{$16 S$ rRNA Gene Sequencing}

The PCR primers 799F-mod6 (5' CMGGATTAGATACCCKGGT$3^{\prime}$ ) and 1114R (5'-GGGTTGCGCTCGTTGC-3') were used to amplify the V5 to V6 hypervariable regions of the $16 \mathrm{~S}$ rRNA genes [14]. The amplification mix contained 5× PrimeSTAR Buffer (Takara Bio Inc., Japan), $2.5 \mathrm{mM}$ concentrations each of deoxynucleotide triphosphates (dNTPs), $2.5 \mathrm{U} / \mu \mathrm{l}$ of PrimeSTAR HS DNA Polymerase, 10 pmol of each primer, and $25 \mathrm{ng}$ of DNA in a reaction volume of $50 \mu \mathrm{l}$. The thermal cycling parameters were as follows: initial denaturation was at $98^{\circ} \mathrm{C}$ for $3 \mathrm{~min}$, followed by 30 cycles of $98^{\circ} \mathrm{C}$ for $10 \mathrm{sec}, 55^{\circ} \mathrm{C}$ for $15 \mathrm{sec}$, and $72^{\circ} \mathrm{C}$ for $30 \mathrm{sec}$, and a final 3-min extension at $72^{\circ} \mathrm{C}$. PCR products were purified using PCR Wizard SV Gel and a PCR Clean-Up System Purification Kit (Promega, USA).

\section{S rRNA Gene Library Preparation and MiSeq Sequencing}

After sample preparation and quality control, 16S rRNA gene amplicons were sequenced using the Illumina MiSeq platform at Macrogen Inc. (Korea) according to the manufacturer's instructions. Briefly, the sequencing library was prepared by random fragmentation of the DNA samples followed by $5^{\prime}$ and $3^{\prime}$ adapter ligation. This step attaches dual indices and Illumina sequencing adapters using the Nextera XT Index Kit. The final products were normalized and pooled using the PicoGreen, and the size of libraries was verified using the TapeStation DNA ScreenTape D1000 (Agilent). The PCR conditions were as follows: initial denaturation $\left(95^{\circ} \mathrm{C}\right.$ for $\left.3 \mathrm{~min}\right) 8$ amplification cycles $\left(95^{\circ} \mathrm{C}\right.$ for $30 \mathrm{sec}, 55^{\circ} \mathrm{C}$ for $30 \mathrm{sec}, 72^{\circ} \mathrm{C}$ for $\left.30 \mathrm{sec}\right)$ and final elongation $\left(72^{\circ} \mathrm{C}\right.$ for $5 \mathrm{~min})[15,16]$. 


\section{S rRNA Gene Sequence Analysis}

The 16S rRNA gene sequences were processed using Mothur software to remove low-quality sequences [17]. Briefly, sequences that did not match the PCR primers were eliminated from demultiplexed sequence reads. We also removed sequences containing ambiguous base calls and sequences with a length less than $100 \mathrm{bp}$ to minimize the effects of random sequencing errors $[18,19]$. Chimeric sequences were further deleted using the UCHIME algorithm implemented in Mothur. Next, the QIIME (Quantitative Insights into Microbial Ecology) software package (version 1.9.1) was used for de novo operational taxonomic unit (OTU) clustering with an OTU definition at an identity cutoff of $97 \%$ [20]. Taxonomic assignment was performed using the naïve Bayesian RDP Classifier and the Greengenes reference database. Microbial alpha diversity indices including Chao1, observed OTUs, Shannon and Simpson indices were computed using QIIME. A two-sided Welch's $t$ test in Statistical Analysis of Metagenomic Profiles (STAMP) software v2.1.3 was used to identify significant differences in relative abundance of microbial taxa of the two groups with $p<0.05$ considered significant [21]. The Analysis of Similarities (ANOSIM) was used to calculate $p$ values and to test differences of beta-diversity between the two groups using QIIME based on the unweighted UniFrac distances. The principal component analysis (PCoA) plots were generated using QIIME based on the unweighted UniFrac distance matrix. Furthermore, linear discriminant analysis (LDA) effect size (LEfSe) was used to reveal differentially abundant bacterial taxa between the animal groups [22]. The LEfSe algorithm emphasizes both statistical and biological relevance for biomarker discovery. A size-effect threshold of 4.0 on the logarithmic LDA score was used for discriminative taxonomic biomarkers. A $p$-value of $<0.05$ was considered significant in Kruskal-Wallis and pairwise Wilcoxon tests, respectively.

\section{Histological Examination}

Two dogs with PVE that died at 8 weeks of age were immediately necropsied, and the jejuna were collected for histological examination. The intestinal tissues were fixed in a $10 \%$ neutral formalin solution before being embedded in paraffin wax and sectioned into $5-\mu \mathrm{m}$ thickness. The sections were deparraffinized, rehydrated, and stained with hematoxylin and eosin solution using standard procedures [23]. The sections were mounted with mounting medium and observed under a light microscope.

\section{Results}

\section{Diagnosis of the Parvovirus Infection in Beagle Puppies}

All the puppies were healthy at weaning at 4 weeks of age. However, at week 6, dogs naturally infected with CPV began to show clinical signs, including anorexia, vomiting, and diarrhea. The CPV infection was confirmed at week 6 by detecting parvovirus antigen in feces using a commercial ELISA kit, the SNAP Parvo Test (IDEXX Laboratories, USA). The CPV-infected puppies showed significantly higher temperature $\left(40.87 \pm 0.64\right.$ vs $\left.38.67 \pm 0.09^{\circ} \mathrm{C}\right)$, pulse rate $(221.00 \pm 6.83$ vs $176.00 \pm 7.30 \mathrm{bpm})$, and respiratory rate $(69.00 \pm 3.83$ vs $31.00 \pm 3.83 \mathrm{bpm})$ compared to control puppies $(p<0.05)$ (Table 3$)$. White blood cell numbers were significantly lower in the CPV group than control group (WBC, $2.65 \pm 2.61$ vs $\left.8.25 \pm 0.83 \times 10^{9} / \mathrm{L}\right)(p<0.05)$. Serum chemistry showed that total protein $(3.27 \pm 2.49$ vs $4.80 \pm 0.25)$ and albumin $(1.77 \pm 0.49$ vs $2.67 \pm 0.09)$ were significantly lower in the CPV group compared to the control group $(p<0.05)$ (Table 4$)$. The vital signs, electrolytes, complete blood count analysis and body weight of each individual dog are provided in the supplementary materials (Table S2-S6). Ultrasonography revealed that all puppies with PVE had a thickened intestine, a small amount of ascites fluid, and fluid stored in the intestines.

\section{Intestinal Histology of Dogs with Canine Parvovirus Infection}

Two dogs with severe clinical signs of CPV infection died at week 8. A histological examination was conducted using the small intestinal tissue of the diseased dogs. Histological

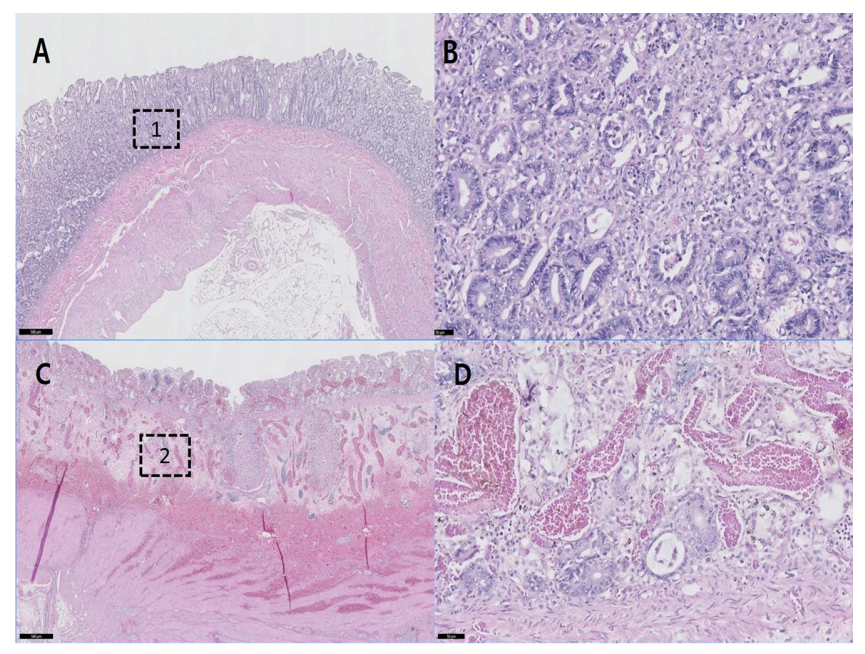

Fig. 1. Intestinal morphology of dogs with canine parvoviral enteritis.

(A, C) Depicts the histology of the small intestine at magnification of 40X; (B, D) photographs of the small intestine at magnification of 100X. Numbers 1 and 2 in photographs $(\mathbf{A}, \mathbf{C})$ indicate the magnified regions at 100X as shown in photographs $(\mathbf{B}, \mathbf{D})$, respectively. The diffusely collapsed small intestinal mucosal layer in two dogs with CPV can be identified (A, C). Small numbers of lymphocytes and plasma cells were identified in the lamina propria (B), and the intestinal tissue was congested with red blood cells (D). 
Table 1. Alpha diversity indices of gut microbial community in healthy dogs and dogs with CPV infection at different age in weeks.

\begin{tabular}{ccccccccc}
\hline \multirow{2}{*}{ Week } & \multicolumn{2}{c}{ No. of observed OTUs } & \multicolumn{2}{c}{ Chao1 } & \multicolumn{2}{c}{ Shannon } & \multicolumn{2}{c}{ Simpson } \\
\cline { 2 - 10 } & Control & CPV & Control & CPV & Control & CPV & Control & CPV \\
\hline Week 4 & $1,476.25 \pm 479.79$ & $1655 \pm 426.98$ & $3,404.66 \pm 1,455.51$ & $3,760.51 \pm 1,161.19$ & $4.77 \pm 0.59$ & $4.99 \pm 0.65$ & $0.89 \pm 0.04$ & $0.91 \pm 0.04$ \\
Week 6 & $1,465.25 \pm 120.96$ & $1,002.25 \pm 347.09$ & $3,333.25 \pm 214.34^{\mathrm{a}}$ & $2,111.26 \pm 717.52$ & $4.64 \pm 0.30$ & $3.83 \pm 0.73$ & $0.88 \pm 0.02$ & $0.83 \pm 0.06$ \\
Week 8 & $714 \pm 251.22$ & $742 \pm 118.79$ & $1539.00 \pm 591.21$ & $1,835.90 \pm 808.25$ & $3.51 \pm 0.52$ & $3.17 \pm 0.26$ & $0.84 \pm 0.02$ & $0.81 \pm 0.04$ \\
Week 12 & $613 \pm 442.95$ & $820.5 \pm 211.42$ & $1,386.18 \pm 1012.24$ & $1,777.70 \pm 193.31$ & $2.73 \pm 1.15$ & $3.52 \pm 0.55$ & $0.68 \pm 0.23$ & $0.80 \pm 0.02$ \\
\hline
\end{tabular}

Canine parvoviral infection decreased the microbial diversity in dogs.

${ }^{a}$ Significantly different with $p<0.05$; Values are presented as mean \pm SD

examination revealed that the small intestine mucosa was diffusely collapsed. Normal villi and cryptal mucosal structures were rarely identified. The villous structure was diffusely collapsed and fragmented and had hypereosinophilic homogenous cytoplasm with karyolytic, karyorrhectic, and pyknotic debris. The crypts were diffusely collapsed, necrotic, hemorrhagic, and destroyed. The lamina propria was collapsed and disorganized with small numbers of lymphocytes, eosinophils, and neutrophils. Submucosal lymphoid follicles were also necrotic (Fig. 1).

\section{Sequence Analysis}

The $16 \mathrm{~S}$ rRNA gene sequencing produced a total of $5,281,451$ sequence reads from all the fecal samples $(n=26$, mean $\pm S D=203,132 \pm 11,235)$. After quality filtering, 3,575,999 sequence reads were obtained from the fecal samples of which 1,843,982 and 1,732,017 sequences were obtained from the healthy and CPV group, respectively. For alpha and beta diversity analyses, all samples were rarefied at the minimum number of sequences $(114,960)$ to account for unequal sequencing depth (Table S1). All raw 16S rRNA gene data used in this study were deposited in the National Center for Biotechnology Information (NCBI) under Sequence Read Archive (SRA), accession number PRJNA474706.

\section{Alpha Diversity}

The alpha diversity analysis was performed to compare the number of observed OTUs, Chao1, Shannon, and Simpson diversity indices between healthy dogs and dogs with CPV at different weeks of age [24] (Table 1). The alpha diversity indices were not significantly different between the control and CPV-infected groups before and after the CPV infection. However, the CPV-infected group showed lower alpha diversity indices compared to the control group at 6 weeks of age when the CPV-infected group was diagnosed as PVE. Chao1, a measure of species richness, was significantly higher in healthy dogs than in the CPVinfected group at 6 weeks of age, the time that CPV was detected $(p<0.05)$. These results suggest that CPV infection causes dysbiosis in the intestinal microbial community and that it decreases the diversity of microbes in the intestine of dogs.

\section{Ecological Succession of the Gut Microbiota in Healthy Dogs and Dogs Naturally Infected with Canine Parvovirus}

The longitudinal changes in the gut microbiota of healthy dogs compared to those of dogs with CPV before and after infection are shown in Fig. 2A. Regardless of the health status, the microbiota of all dogs were composed of

Table 2. Bacterial phyla present at $>1 \%$ mean relative abundance in healthy dogs and dogs with CPV infection.

\begin{tabular}{|c|c|c|c|c|c|c|}
\hline \multirow{2}{*}{ Taxon } & \multicolumn{2}{|c|}{ Healthy $(n=4)$} & \multicolumn{2}{|c|}{$\mathrm{CPV}(n=4)$} & \multirow[b]{2}{*}{$p$-value } & \multirow[b]{2}{*}{$q$-value ${ }^{a}$} \\
\hline & mean \% & $\mathrm{SD} \%$ & mean \% & $\mathrm{SD} \%$ & & \\
\hline Proteobacteria ${ }^{* * *}$ & 27.29 & 9.37 & 67.90 & 25.36 & $<0.001$ & $<0.001$ \\
\hline Firmicutes & 27.17 & 8.68 & 19.53 & 12.73 & 0.179 & 0.402 \\
\hline Bacteroidetes $^{* * *}$ & 26.79 & 3.62 & 6.71 & 8.81 & $<0.001$ & 0.003 \\
\hline Fusobacteria* & 16.16 & 3.89 & 3.78 & 7.13 & 0.031 & 0.094 \\
\hline Actinobacteria & 2.15 & 0.80 & 1.75 & 2.06 & 0.943 & $>0.999$ \\
\hline
\end{tabular}

Comparison of the mean relative abundance and standard deviation of the most abundant bacterial groups based on sequencing of the 16S rRNA gene between healthy and diseased dogs at week 6 at the time of canine parvovirus diagnosis.

${ }^{\mathrm{a}} q$-values adjusted based on the Benjamini \& Hochberg False discovery rate; ${ }^{* * *} p<0.001,{ }^{*} p<0.05$. 


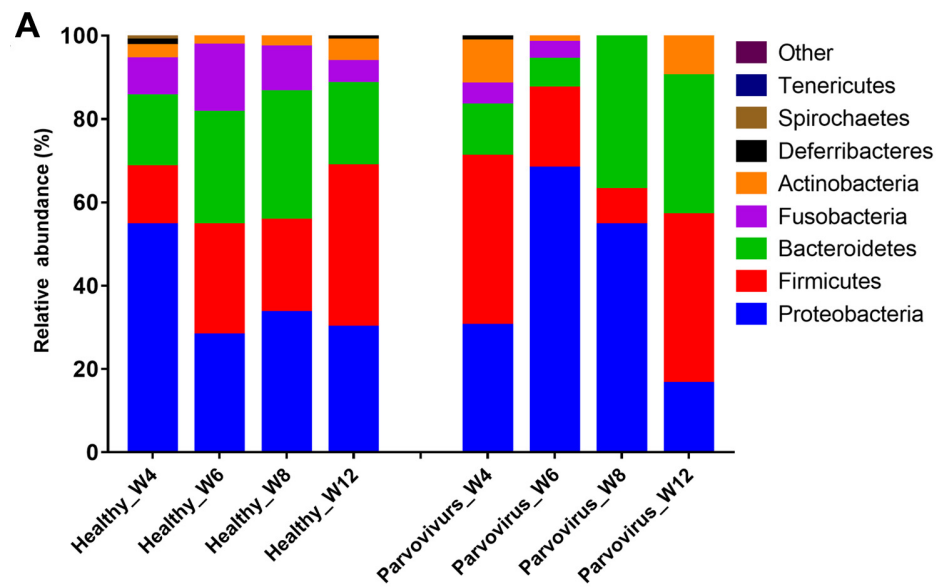

\section{B}

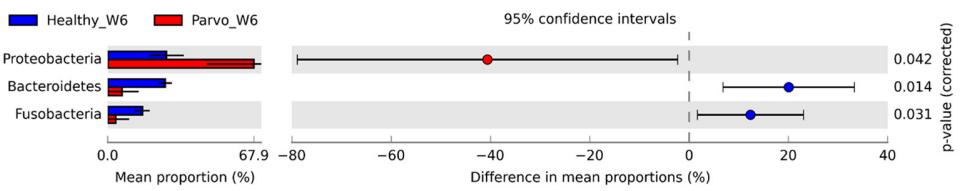

C

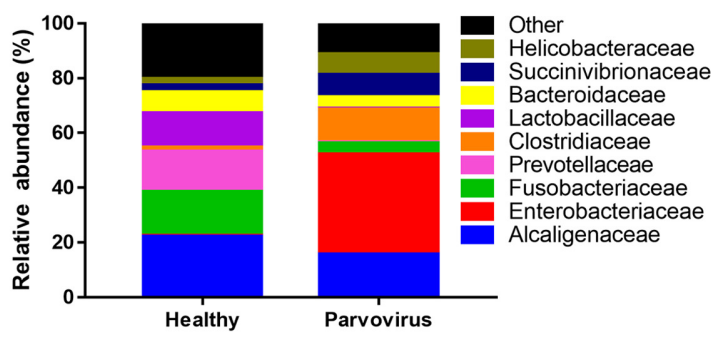

D

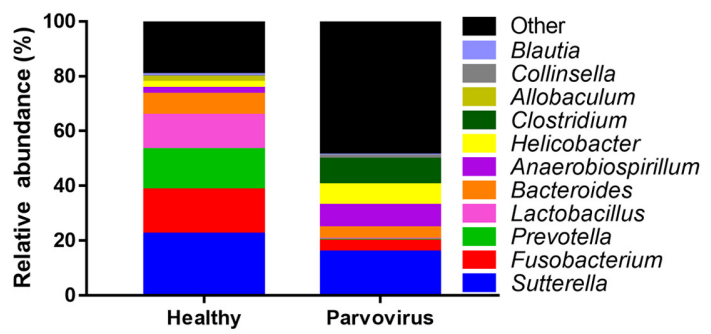

Fig. 2. Compositions of the intestinal microbiota of healthy dogs and dogs with canine parvoviral enteritis.

(A) Longitudinal changes in the composition of microbiota at the phylum level between healthy dogs and dogs with CPV enteritis. The letter W denotes week and numbers denote age in weeks. (B) Extended-error plot identifying significant differences in the relative abundance of bacteria at the phylum level at 6 weeks of age between healthy and diseased dogs. The differences in the proportions of bacteria were tested using a twosided Welch's $t$-test, and a $p<0.05$ was considered significant. The classification of the sequences at the family (C) and genus (D) levels between healthy and diseased dogs at the time of diagnosis of CPV enteritis at 6 weeks of age.

bacteria belonging to phyla Proteobacteria, Firmicutes, Bacteroidetes, Fusobacteria and Actinobacteria, which collectively accounted for up to $99 \%$ of the total relative abundance (Fig. 2A and Table 2). In the healthy dogs, the relative abundance of Proteobacteria and Fusobacteria was substantially decreased while the population of Firmicutes and Actinobacteria increased as the dogs aged. However, the composition of the beagle gut microbiota experienced a clear change upon infection with CPV. Contrary to the microbial succession in normal beagles, dogs infected with CPV had a substantial increase in the relative abundance of the phylum Proteobacteria and a decrease in the phyla

Table 3. Vital signs of dogs at the time of parvoviral enteritis diagnosis at 6 weeks of age.

\begin{tabular}{lccc}
\hline & Healthy $(n=4)$ & $\mathrm{CPV}(n=4)$ & $p$ value $^{\mathrm{a}}$ \\
\hline Body weight $(\mathrm{kg})$ & $1.98 \pm 0.21$ & $2.07 \pm 0.10$ & 0.886 \\
Temperature $\left({ }^{\circ} \mathrm{C}\right)^{*}$ & $38.67 \pm 0.09$ & $40.87 \pm 0.64$ & 0.029 \\
Pulse rate $(\mathrm{bpm})^{*}$ & $176.00 \pm 7.30$ & $221.00 \pm 6.83$ & 0.029 \\
Respiratory rate $(\mathrm{bpm})^{*}$ & $31.00 \pm 3.83$ & $69.00 \pm 3.83$ & 0.029 \\
\hline
\end{tabular}

${ }^{\text {a }}$ The $p$-values were measured by Mann-Whitney test $;{ }^{*} p<0.05$.
Firmicutes, Bacteroidetes, and Actinobacteria at 6 weeks of age (the time of PVE diagnosis). When puppies recovered from CPV infection at 12 weeks of age, there was a transition back to a normal microflora composition similar to that of healthy dogs. The proportion of Proteobacteria decreased whereas Firmicutes and Actinobacteria increased as puppies recovered from CPV infection at 12 weeks of age (Fig. 2).

We utilized an extended error plot generated in STAMP to visualize the differences in gut microbial community composition between healthy dogs and dogs with CPV at each time point. At 4 weeks of age, the relative abundance of each taxon was similar, and there were no significant differences observed between the two groups. (Fig. 2A). However, major differences of the gut microbial compositions between groups were observed at 6 weeks of age (Fig. 2B). We found that the relative abundance of Proteobacteria was significantly higher $(p=0.042)$ in dogs with CPV than those in healthy dogs. In addition, dogs with CPV had significantly lower relative abundance of the phyla Bacteroidetes and Fusobacteria $(p<0.05)$ compared to the healthy dogs at 6 weeks of age (Fig. 2B and Table 2). 
Table 4. Comparison of complete blood count, serum chemistry and electrolyte analysis between healthy dogs and dogs with CPV infection at 6 weeks of age.

\begin{tabular}{|c|c|c|c|}
\hline & $\begin{array}{c}\text { CPV } \\
(n=4)\end{array}$ & $\begin{array}{l}\text { Healthy } \\
(n=4)\end{array}$ & $p$-value ${ }^{\mathrm{a}}$ \\
\hline \multicolumn{4}{|l|}{ Complete blood count } \\
\hline PCV $(\%)$ & $32.60 \pm 3.69$ & $36.82 \pm 2.36$ & 0.200 \\
\hline $\mathrm{WBC}(10 \times 9 / \mathrm{L})$ & $2.65 \pm 2.61$ & $8.25 \pm 0.83$ & 0.029 \\
\hline Neutrophil $(10 \times 9 / \mathrm{L})$ & $1.75 \pm 1.81$ & $5.72 \pm 0.51$ & 0.029 \\
\hline Lymphocyte $(10 \times 9 / \mathrm{L})$ & $0.57 \pm 0.49$ & $1.67 \pm 0.38$ & 0.029 \\
\hline Monocyte $(10 \times 9 / \mathrm{L})$ & $0.35 \pm 0.07$ & $0.67 \pm 0.26$ & 0.267 \\
\hline Eosinophil $(10 \times 9 / \mathrm{L})$ & $0.20 \pm 0.10$ & $0.17 \pm 0.05$ & 0.857 \\
\hline $\operatorname{PLT}(10 \times 9 / \mathrm{L})$ & $142.25 \pm 89.55$ & $277.50 \pm 21.733$ & 0.029 \\
\hline \multicolumn{4}{|l|}{ Serum chemistry analysis } \\
\hline AST (U/L) & $46.00 \pm 32.62$ & $21.25 \pm 7.32$ & 0.686 \\
\hline $\operatorname{ALT}(\mathrm{U} / \mathrm{L})$ & $264.75 \pm 217.33$ & $57.75 \pm 5.43$ & 0.343 \\
\hline $\operatorname{ALKP}(\mathrm{U} / \mathrm{L})$ & $610.00 \pm 63.21$ & $298.25 \pm 43.95$ & 0.029 \\
\hline GGT (U/L) & $9.00 \pm 1.82$ & $2.75 \pm 0.95$ & 0.029 \\
\hline Tbil (mg/dL) & $0.65 \pm 0.64$ & 0 & 0.029 \\
\hline $\mathrm{TP}(\mathrm{g} / \mathrm{dL})$ & $3.27 \pm 2.49$ & $4.80 \pm 0.25$ & 0.029 \\
\hline $\operatorname{ALB}(\mathrm{g} / \mathrm{dL})$ & $1.77 \pm 0.49$ & $2.67 \pm 0.09$ & 0.029 \\
\hline Crea $(\mathrm{mg} / \mathrm{dL})$ & $0.25 \pm 0.12$ & $0.25 \pm 0.05$ & 1.000 \\
\hline BUN (mg/dL) & $4.62 \pm 0.77$ & $9.77 \pm 1.21$ & 0.029 \\
\hline Glucose (mg/dL) & $107.50 \pm 12.55$ & $121.50 \pm 20.80$ & 0.486 \\
\hline $\mathrm{LIP}(\mathrm{U} / \mathrm{L})$ & $10.17 \pm 2.75$ & $11.62 \pm 1.94$ & 0.486 \\
\hline $\mathrm{IP}(\mathrm{mg} / \mathrm{dL})$ & $4.27 \pm 0.92$ & $3.90 \pm 0.53$ & 0.686 \\
\hline Amylase (U/L) & $308.25 \pm 71.07$ & $303.50 \pm 63.77$ & 1.000 \\
\hline Chole (mg/dL) & $190.50 \pm 50.35$ & $196.50 \pm 17.40$ & 0.886 \\
\hline \multicolumn{4}{|l|}{ Electrolyte analysis } \\
\hline $\mathrm{Na}^{+}(\mathrm{mmol} / \mathrm{L})$ & $138.97 \pm 3.07$ & $147.40 \pm 1.29$ & 0.029 \\
\hline $\mathrm{K}^{+}(\mathrm{mmol} / \mathrm{L})$ & $3.12 \pm 0.19$ & $3.56 \pm 0.09$ & 0.029 \\
\hline $\mathrm{Cl}^{-}(\mathrm{mmol} / \mathrm{L})$ & $104.87 \pm 4.68$ & $109.42 \pm 2.87$ & 0.200 \\
\hline
\end{tabular}

PCV (packed cell volume), WBC (white blood cell), PLT (platelet), AST (aspartate aminotransferase), ALT (alanine aminotransferase), ALKP (alkaline phosphatase), GGT (gamma-glutamyl transferase), Tbil (total bilirubin), TP (total protein), ALB (albumin), Crea (creatinine), BUN (blood urea nitrogen), LIP (lipase), IP (phosphorusinorganic), Chole (cholesterol), $\mathrm{Na}^{+}$(sodium ion), $\mathrm{K}^{+}$(potassium ion), $\mathrm{Cl}^{-}$(chloride ion)

${ }^{a}$ The $p$-values were measured by Mann-Whitney test.

At the family level, the gut microbiota of healthy dogs at 6 weeks of age were predominantly composed of Alcaligenaceae $(22.22 \%)$, Fusobacteriaceae $(16.12 \%)$, Prevotellaceae $(14.63 \%)$ and Lactobacillaceae (12.69\%). Enterobacteriaceae in dogs with PVE was most abundant accounting for $36.44 \%$ of the total bacterial population, and it was significantly higher than in healthy puppies $(0.21 \%)(p<0.05)$ (Fig. $2 \mathrm{C}$ and Fig. S1).

At the genus level, the gut microbiota of healthy dogs at 6 weeks of age was primarily composed of Sutterella $(21 \%)$, Fusobacterium (16.14\%), Prevotella (14.26\%), Lactobacillus
(13.08\%) and Bacteroides with an overall relative abundance of $73.08 \%$ of the total taxa. By comparison, the gut microbiota of dogs with CPV were composed of bacteria belonging to genera Sutterella (15.54\%), Clostridium $(9.07 \%)$, Anaerobiospirillum (8.06\%) and Helicobacter (7.75\%) (Fig. 2D and Fig. S1). The relative abundances of Prevotella and Lactobacillus in healthy puppies were significantly higher than in dogs with CPV infection $(p<0.05)$.

The microbiota of individual dogs and their relative abundance at the phylum (Fig. S2), family (Fig. S3) and genus (Fig. S4) levels were shown in the supplementary materials. 


\section{Differences in Bacterial Composition between Healthy and CPV-Infected Dogs}

The linear discriminant analysis (LDA) effect size (LEfSe) method was used to determine differentially abundant bacterial taxa among both CPV-infected and healthy dog groups. The LEfSe algorithm determines the bacterial taxa that are most likely to explain the differences between the two groups by implementing standard statistical tests to predict potential biomarkers with biological and statistical significance. A total of 9 bacterial groups were differentially enriched between healthy dogs and dogs with CPV with a $p$-value $<0.05$ and LDA score $>4.0$. The plot from LEfSe analysis shows the LDA scores of microbial taxa with significant differences between the two groups (Fig. S5). The cladogram showed differences in 6 taxa between the healthy and CPV groups (Fig. S6). At the phylum level, Bacteroidetes and Proteobacteria showed significant differences in relative abundance between healthy and CPV-infected dogs. At the family level, the differentially abundant taxa that can be considered as potential biomarkers showing significant differences between healthy and CPVinfected dogs were Prevotellaceae, Lactobacillaceae and Enterobacteriaceae. At the genus level, the biomarkers showing significant differences between the two groups were Lactobacillus and Prevotella.

\section{Beta Diversity}

A principal component analysis (PCoA) based on the unweighted UniFrac distance comparisons was performed to characterize the global differences in the intestinal microbial communities between the healthy and CPV groups. The PCoA plot showed a tight clustering of microbiomes from healthy dogs which indicates microbiome stability; while those dogs with CPV showed a greater dispersion of microbial community composition than the control (Fig. S7). The PCoA plot showed significant separation between the samples from healthy dogs and dogs with CPV based on analysis of similarities (ANOSIM). We used the ANOSIM of unweighted UniFrac distance metrics to statistically compare the bacterial communities of healthy dogs and diseased dogs at different time points. During the time of diagnosis of CPV at week 6, the ANOSIM of unweighted UniFrac distance metrics indicated that the intestinal microbiota of healthy dogs and that of dogs with CPV were significantly different $(p=0.029)$ with an $R$-value of 0.730 , demonstrating that there was a difference in the gut microbial communities between the two groups. At 8 weeks and 12 weeks of age, the ANOSIM of unweighted UniFrac distance metrics between healthy dogs and dogs that recovered from CPV were not significantly different $(p>0.05)$. These findings suggest that CPV explained the variation in the microbial composition between healthy dogs and dogs with CPV.

\section{Discussion}

It is important to understand virus-bacterial interactions because microbiota can influence viral infection and vice versa. Even though CPV is the major cause of hemorrhagic gastroenteritis and myocarditis in puppies [25], there was a lack of information regarding parvovirus-bacterial interactions. Therefore, the aim of this study was to evaluate the gut microbial shifts in dogs naturally infected with CPV. To our knowledge, this is the first study to evaluate the gut microbiota of dogs with CPV before and after infection compared to healthy dogs. In this study, we found no significant differences in overall species richness and diversity between healthy dogs and dogs with CPV at different time points. However, CPV infection decreased overall diversity indices. In addition, we found significant differences in the microbial community structure and membership indicating that the gut microbiota differs between healthy dogs and dogs with CPV. Generally, the impact of viral infections on host microbiota remains poorly understood. However, recent studies demonstrated the consequences of viral infections in the intestinal microbiota. For example, Yildiz et al. [26] showed that Influenza A virus (IAV) infection impacts the microbiota dynamics and causes dysbiosis in the small intestine in a mouse model using culture-dependent and cultureindependent techniques [26]. In a more recent study, it has been suggested that viruses have the ability to shape the community dynamics and function of the host microbiota through lysis of commensal bacteria leading to prophage induction that could potentially facilitate colonization by pathogenic bacteria [27].

The number of observed OTUs was associated with the stages of CPV disease progression over time. Before the onset of the infection at week 4 , a similar number of observed OTUs between the two groups was detected. Following infection at week 6, the number of OTUs drastically decreased suggesting that CPV infection causes dysbiosis which then reduced the diversity of microbes in the gut and increased the population of pathogenic bacteria, as we have demonstrated in the taxonomic analysis. The number of observed OTUs between the control and CPV groups seemed to be equivalent at week 8 because the puppies in the CPV group have recovered from the disease. 
However, no significant differences were observed in the number of observed OTUs at week 8 suggesting that the microbiota composition between the two groups were likely to be similar. In addition, it was observed that CPVinfected puppies did not show clinical signs of infection as early as week 7 suggesting that the overall health of the puppies had improved and the microbiota was starting to return to a normal state.

Furthermore, beta diversity analysis using PCoA plots based on the UniFrac distance matrix was used to visualize the similarities or dissimilarities of microbiota distances between healthy and CPV-infected dogs (Fig. S2). The application of different statistical analyses of the bacterial communities allowed us to differentiate the bacterial taxa that altered the microbiota of the CPV-infected dogs. During the time of diagnosis of CPV in dogs, the ANOSIM of UniFrac distance metrics showed that the intestinal microbiota of healthy dogs and dogs with CPV were significantly different $(p=0.029)$ with $R$-value of 0.730 , demonstrating that there was a great difference in the composition of the gut microbial communities between the two groups. From this perspective, it can be proposed that CPV can induce the Anna Karenina Principle (AKP) effect on the microbiome of dogs, which states that 'all healthy microbiomes are similar and each dysbiotic microbiome is dysbiotic in its own way' [28]. When the samples from the two clusters in dogs with CPV were analyzed separately, it was observed that samples before the infection at week 4 were clustered into one, and samples after the infection at week 8 and week 12 were grouped into another cluster. Interestingly, week-6 samples were evenly distributed between the two clusters among CPV-infected dogs. The dispersion of the microbiome essentially reflects the variation of microbiota composition of dogs and their abundance differs from sample to sample among dogs infected with CPV. Several lines of evidence suggest that there is an interaction between canine gut microbiome and diseases such as inflammatory bowel disease (IBD) [29, 30], chronic diarrhea [31] and gastric dilation-volvulus [32] leading to dysbiosis of the gut microbiome. Additionally, we determined the microbial taxa that contributed to the separation of the intestinal microbiota of dogs at the early (W4 and W6) and late stage (W8 and W12) of CPV infection. At the genus level, Anaerobiospirillum and Fusobacterium were significantly more abundant at the early-stage, while Prevotella was significantly more abundant at the late-stage in dogs infected with CPV $(p<0.05)$ (Fig. S8). Our findings can be interpreted as evidence of gut microbiome dysbiosis in response to natural infection of CPV in dogs. Despite this evidence that the intestinal microbiota may play a role in the pathogenesis of PVE, the actual mechanisms of the host-microbe interactions remain elusive; hence, studies that assess the functional aspects of the microbiota are necessary.

In our study, alteration of the gut microbial diversity, called dysbiosis, has been associated with the CPV infection. Dysbiosis is characterized by loss of beneficial microbial organisms, expansion of pathobionts or potentially harmful microorganisms, and loss of overall microbial diversity [33]. In agreement with previous investigations, the healthy gut microbiota of dogs in this study was dominated by phyla Firmicutes, Bacteroidetes, Proteobacteria, Actinobacteria and Fusobacteria bacteria, which constitute approximately $99 \%$ of the gut microbiota in dogs [8, 34]. However, the most noticeable finding in dogs with CPV was the increase in the relative abundance of the phylum Proteobacteria and a decrease in Firmicutes, Bacteroidetes, and Actinobacteria at 6 weeks of age. This finding agrees with a recent study showing an increase in Proteobacteria in dogs with intestinal inflammation [11]. In dogs with canine IBD, the most common microbial changes observed are decreases in the bacterial phyla Firmicutes and Bacteroidetes with a concurrent increase in Proteobacteria [35]. Proteobacteria have been shown to be one of the most abundant phyla in the gastrointestinal tract of dogs [36]. Recently, Proteobacteria have been identified as one of the fecal bacterial markers of inflammation in dogs with acute hemorrhagic diarrhea syndrome [37]. In this study, within the Proteobacteria phylum, we also found that Enterobacteriaceae was significantly enriched in dogs with CPV. Enterobacteriaceae have been linked to the pathogenesis of chronic enteropathies in dogs [38]. Our results agree with studies of humans with intestinal inflammation where similar patterns of dysbiosis were observed. For example, it was shown that humans with Crohn's disease had a decrease in the bacterial phylum Firmicutes and a concurrent increase in the abundance of facultative anaerobes such as Enterobacteriaceae [39]. This finding suggested that Enterobacteriaceae is associated with PVE.

However, there is a limitation to this study that needs to be addressed. The sample size of animals used in the study limited the statistical power, since the dogs were naturally infected with CPV. It has been considered that relative effect sizes of biological replicates can lead to a more robust conclusion and evaluate potentially relevant biological effects associated with the CPV infection. Despite this limitation, we were able to characterize the composition of the intestinal microbiome of puppies naturally infected 
with CPV and demonstrate microbial shifts in response to CPV infection through cross-sectional and longitudinal approaches. In the future, metagenomics, metatranscriptomics and metabolic approaches coupled with culturedependent techniques including isolation of novel canine gut bacteria would broaden our current understanding of the intestinal microbial community structure and function in dogs with CPV infection.

In summary, the present study offers significant insights into the longitudinal changes in the gut microbiota composition in dogs with PVE using high-throughput $16 \mathrm{~S}$ rRNA gene sequencing. Our results showed that CPV was associated with microbial dysbiosis of the intestinal microbiota. Alpha diversity metrics showed decreased species richness and evenness in dogs with CPV infection compared to those in healthy dogs. There were also significant differences in microbial community membership between healthy dogs and CPV-infected dogs. Despite this, the current data are limited to being used to correlate compositional changes with corresponding functional aspects in the pathogenesis of canine PVE, evaluation of the role of Enterobacteriaceae family in the pathophysiology of canine parvovirus disease warrants further study since this family was identified as one of the fecal bacterial biomarkers of inflammation or other diseases in dogs. Nevertheless, we acknowledge that intravenous injection of analgesics and antiemetics, and subcutaneous injection of the G-CSF might have effects on the gut microbiota. However, parenteral administration of non-antibiotic materials should have minimal effects on the gut microbiota.

In addition, co-infection of pathogenic bacteria identified in this study might be attributed to the increase of severity of lesions and clinical signs of CPV infection. Therefore, there is a need for an effective treatment to support the dogs in the early stages of CPV through gut microbiota modulation by targeting the enriched pathogenic bacteria. Although our findings have provided insights into the pathology of CPV infection which can be used for treatment of CPV infection, further experimental and clinical studies are needed to establish the importance of these microbiota and its interaction with CPV.

\section{Acknowledgments}

We used bioinformatics analysis tools and methods developed by the fund (Project No. PJ01261501), Rural Development Administration, Republic of Korea and the Individual Basic Science \& Engineering Research Program (No. 2019R1F1A1063306) through the National Research
Foundation of Korea (NRF) funded by the Ministry of Education. We thank Mo Re Kim (Westborough High School) for the English grammar corrections.

\section{Conflict of interest}

The authors confirm that they have no conflicts of interest.

\section{References}

1. Nandi S, Kumar M. 2010. Canine parvovirus: current perspective. Indian J. Virol. 21: 31-44.

2. Kilian E, Suchodolski JS, Hartmann K, Mueller RS, Wess G, Unterer S. 2018. Long-term effects of canine parvovirus infection in dogs. PLoS One 13: e0192198.

3. Sime TA, Powell LL, Schildt JC, Olson EJ. 2015. Parvoviral myocarditis in a 5-week-old Dachshund. J. Vet. Emerg. Crit. Care (San Antonio) 25: 765-769.

4. Tress B, Dorn ES, Suchodolski JS, Nisar T, Ravindran P, Weber $\mathrm{K}$, et al. 2017. Bacterial microbiome of the nose of healthy dogs and dogs with nasal disease. PLoS One 12: e0176736.

5. Guevarra RB, Lee JH, Lee SH, Seok MJ, Kim DW, Kang BN, et al. 2019. Piglet gut microbial shifts early in life: causes and effects. J. Anim. Sci. Biotechnol. 10: 1.

6. Song ES, Jung SI, Park HJ, Seo KW, Son JH, Hong S, et al. 2016. Comparison of Fecal Microbiota between German Holstein Dairy Cows with and without Left-Sided Displacement of the Abomasum. J. Clin. Microbiol. 54: 1140-1143.

7. Kim HB, Isaacson RE. 2017. Salmonella in Swine: Microbiota Interactions. Ann. Rev. Anim. Biosci. 5: 43-63.

8. Handl S, Dowd SE, Garcia-Mazcorro JF, Steiner JM, Suchodolski JS. 2011. Massive parallel 16S rRNA gene pyrosequencing reveals highly diverse fecal bacterial and fungal communities in healthy dogs and cats. FEMS Microbiol. Ecol. 76: 301-310.

9. Hooda S, Minamoto Y, Suchodolski JS, Swanson KS. 2012. Current state of knowledge: the canine gastrointestinal microbiome. Anim. Health Res. Rev. 13: 78-88.

10. Omori M, Maeda S, Igarashi H, Ohno K, Sakai K, Yonezawa $\mathrm{T}$, et al. 2017. Fecal microbiome in dogs with inflammatory bowel disease and intestinal lymphoma. J. Vet. Med. Sci. 79: 1840-1847.

11. Kalenyak K, Isaiah A, Heilmann RM, Suchodolski JS, Burgener IA. 2018. Comparison of the intestinal mucosal microbiota in dogs diagnosed with idiopathic inflammatory bowel disease and dogs with food-responsive diarrhea before and after treatment. FEMS Microbiol. Ecol. 94(2). doi: 10.1093/femsec/fix173.

12. Song YG, Guevarra RB, Lee JH, Wattanaphansak S, Kang $\mathrm{BN}$, Kim HB, et al. 2017. Comparative analysis of the 
reproductive tract microbial communities in female dogs with and without pyometra through the 16S rRNA gene pyrosequencing. Jpn. J. Vet. Res. 65: 193-200.

13. Guevarra RB, Hong $\mathrm{SH}$, Cho JH, Kim BR, Shin J, Lee JH, et al. 2018. The dynamics of the piglet gut microbiome during the weaning transition in association with health and nutrition. J. Anim. Sci. Biotechnol. 9: 54.

14. Hanshew AS, Mason CJ, Raffa KF, Currie CR. 2013. Minimization of chloroplast contamination in 16S rRNA gene pyrosequencing of insect herbivore bacterial communities. J. Microbiol. Methods 95: 149-155.

15. Kim TY, Lee JJ, Kim BS, Choi SH. 2017. Whole-Body Microbiota of Sea Cucumber (Apostichopus japonicus) from South Korea for Improved Seafood Management. J. Microbiol. Biotechnol. 27: 1753-1762.

16. Choi S, Hwang YJ, Shin MJ, Yi H. 2017. Difference in the Gut Microbiome between Ovariectomy-Induced Obesity and Diet-Induced Obesity. J. Microbiol. Biotechnol. 27: 2228-2236.

17. Schloss PD, Westcott SL, Ryabin T, Hall JR, Hartmann M, Hollister EB, et al. 2009. Introducing mothur: Open-Source, Platform-Independent, Community-Supported Software for Describing and Comparing Microbial Communities. Appl. Environ. Microbiol. 75: 7537-7541.

18. Kim D, Hong S, Kim YT, Ryu S, Kim HB, Lee JH. 2018. Metagenomic Approach to Identifying Foodborne Pathogens on Chinese Cabbage. J. Microbiol. Biotechnol. 28: 227-235.

19. Kim D, Hong S, Na H, Chun J, Guevarra RB, Kim YT, et al. 2018. Analysis of Microbiota in Bellflower Root, Platycodon grandiflorum, Obtained from South Korea. J. Microbiol. Biotechnol. 28: 551-560.

20. Caporaso JG, Kuczynski J, Stombaugh J, Bittinger K, Bushman FD, Costello EK, et al. 2010. QIIME allows analysis of high-throughput community sequencing data. Nature Methods 7: 335-336.

21. Parks DH, Tyson GW, Hugenholtz P, Beiko RG. 2014. STAMP: statistical analysis of taxonomic and functional profiles. Bioinformatics 30: 3123-3124.

22. Segata N, Izard J, Waldron L, Gevers D, Miropolsky L, Garrett WS, et al. 2011. Metagenomic biomarker discovery and explanation. Genome Biol. 12(6): R60.

23. Luna LG. 1968. Manual of histologic staining methods of the Armed Forces Institute of Pathology. pp. 158-160. 3rd Edition. New York: McGraw-Hill.

24. Kim BR, Shin J, Guevarra R, Lee JH, Kim DW, Seol KH, et al. 2017. Deciphering Diversity Indices for a Better Understanding of Microbial Communities. J. Microbiol. Biotechnol. 27: 20892093.

25. Moon HS, Lee SA, Lee SG, Choi R, Jeoung SY, Kim D, et al. 2008. Comparison of the pathogenicity in three different Korean canine parvovirus 2 (CPV-2) isolates. Vet. Microbiol. 131: 47-56.
26. Yildiz S, Mazel-Sanchez B, Kandasamy M, Manicassamy B, Schmolke M. 2018. Influenza A virus infection impacts systemic microbiota dynamics and causes quantitative enteric dysbiosis. Microbiome 6(1): 9.

27. Rowan-Nash AD, Korry BJ, Mylonakis E, Belenky P. 2019. Cross-Domain and Viral Interactions in the Microbiome Microbiol. Mol. Biol. Rev. 83(1). pii: e00044-18.

28. Zaneveld JR, McMinds R, Vega Thurber R. 2017. Stress and stability: applying the Anna Karenina principle to animal microbiomes. Nat. Microbiol. 2: 17121.

29. Vazquez-Baeza Y, Hyde ER, Suchodolski JS, Knight R. 2016. Dog and human inflammatory bowel disease rely on overlapping yet distinct dysbiosis networks. Nat. Microbiol. 1: 16177.

30. Suchodolski JS, Markel ME, Garcia-Mazcorro JF, Unterer S, Heilmann RM, Dowd SE, et al. 2012. The fecal microbiome in dogs with acute diarrhea and idiopathic inflammatory bowel disease. PLoS One 7: e51907.

31. Jia J, Frantz N, Khoo C, Gibson GR, Rastall RA, McCartney AL. 2010. Investigation of the faecal microbiota associated with canine chronic diarrhoea. FEMS Microbiol. Ecol. 71: 304312.

32. Hullar MAJ, Lampe JW, Torok-Storb BJ, Harkey MA. 2018. The canine gut microbiome is associated with higher risk of gastric dilatation-volvulus and high risk genetic variants of the immune system. PLoS One 13: e0197686.

33. Petersen C, Round JL. 2014. Defining dysbiosis and its influence on host immunity and disease. Cell Microbiol. 16: 1024-1033.

34. Suchodolski JS, Dowd SE, Wilke V, Steiner JM, Jergens AE. 2012. 16S rRNA gene pyrosequencing reveals bacterial dysbiosis in the duodenum of dogs with idiopathic inflammatory bowel disease. PLoS One 7: e39333.

35. Guard BC, Suchodolski JS. 2016. HORSE SPECIES SYMPOSIUM: Canine intestinal microbiology and metagenomics: From phylogeny to function. J. Anim. Sci. 94: 2247-2261.

36. Suchodolski JS, Camacho J, Steiner JM. 2008. Analysis of bacterial diversity in the canine duodenum, jejunum, ileum, and colon by comparative $16 \mathrm{~S}$ rRNA gene analysis. FEMS Microbiol. Ecol. 66: 567-578.

37. Heilmann RM, Guard MM, Steiner JM, Suchodolski JS, Unterer S. 2017. Fecal markers of inflammation, protein loss, and microbial changes in dogs with the acute hemorrhagic diarrhea syndrome (AHDS). J. Vet. Emerg. Crit. Care (San Antonio) 27: 586-589.

38. Cassmann E, White R, Atherly T, Wang C, Sun Y, Khoda S, et al. 2016. Alterations of the Ileal and Colonic Mucosal Microbiota in Canine Chronic Enteropathies. PLoS One 11: e0147321.

39. Carding S, Verbeke K, Vipond DT, Corfe BM, Owen LJ. 2015. Dysbiosis of the gut microbiota in disease. Microb. Ecol. Health Dis. 26: 26191. 